\title{
Development of Macro-Cantilever Capacitive Sensor for the Detection of Damages in Civil Engineering Structures
}

\author{
Meghayu Adhvaryu ${ }^{1}$, Piyush N. Patel ${ }^{1}$, Chetan D. Modhera ${ }^{2}$ \\ ${ }^{1}$ Sensor Research Lab, Electronics Engineering Department, Sardar Vallabhbhai National Institute of Technology, Surat \\ 395007, Gujarat, India \\ ${ }^{2}$ Civil, Engineering Department, Sardar Vallabhbhai National Institute of Technology, Surat 395007, Gujarat, India
}

\begin{abstract}
A modified Macro-cantilever capacitive sensor design is presented and used in the development of vibration sensor for Structural Health Monitoring (SHM). The design of sensor consists of parallel feed electrode with integrated support. The overall size of the unit-cell structure is $\mathbf{5 0} \times \mathbf{2 5 1 0}$ mm3. Different civil engineering blocks were tested using the proposed sensor and a comprehensive vibration analysis is presented based on the two dimensional force applied to blocks. The sensor offers a lowest average sensitivity of $0.21631 \mathrm{pF} / \mathrm{mm} 3$. The proposed flex-sensor can also be potentially used for the detection and monitoring of other structural parameters like stress, strain, vibration, load capacity etc.
\end{abstract}

Keywords- Macro-cantilever sensor, Capacitive sensor for vibration analysis, Stress detection, civil engineering block

\section{INTRODUCTION}

Minor deformation can lead to major failures in the civil and mechanical engineering structures like bridges, buildings, air crafts etc. These Structures have multiple important or critical locations. Therefore, wireless, and remote monitoring of crack formation is important in order to access the structural health and its integrity. Various physical parameters like strain, crack, moisture, and corrosion are targeted for Structural Health Monitoring (SHM) [1,2]. Micro-strain sensors with optical fiber [3], electrical resistance electrode [4], piezoelectric transducers [5] and planar capacitive interdigitated electrode (IDE) [68] have been developed for strain sensing .Fiber-based sensor probes offer the highest sensitivity related to the changes in moisture content, corrosion in steel rebar's and structural deformities. But strain sensors are very costly because of its difficult embedded methodology. Furthermore, because of the perturbation of probe adjustments during the embedding process, optical sensors always have measurement errors. Piezoelectric transduces has widely been reported for SHM applications. Although Data received from the acoustic signal is somewhat unreliable.

In this work scaled up design of micro-cantilever sensor [9] is utilized and fabricated. It will be utilized to understand load and vibrations analogy on civil structures and corresponding effect on structural failures.

\section{DEVELOPMENT OF PLANNER MACRO CANTILEVER SENSOR}

\section{A. Design \& Fabrication of Macro Cantilever Unit Cell} Sensor

The capacitive sensors can also be modelled for vibration analysis in civil engineering structures. For this, macro cantilever arrangement is required. The design of proposed macro cantilever is represented in Fig. The cantilever is modelled using a foam-based substrate of relative permittivity $\varepsilon_{\mathrm{r}}=2.2$ and thickness of $2 \mathrm{~mm}$. The selection of flexible substrate is required so that transverse acting force can bend the positive cantilever. Moreover, large vibration dents damages the sensor plate.

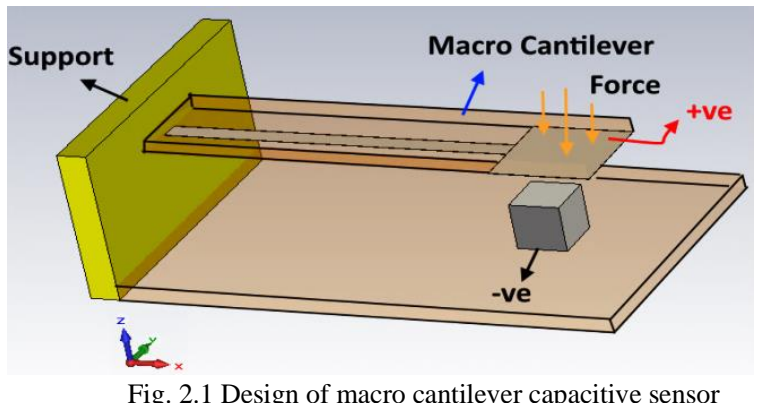

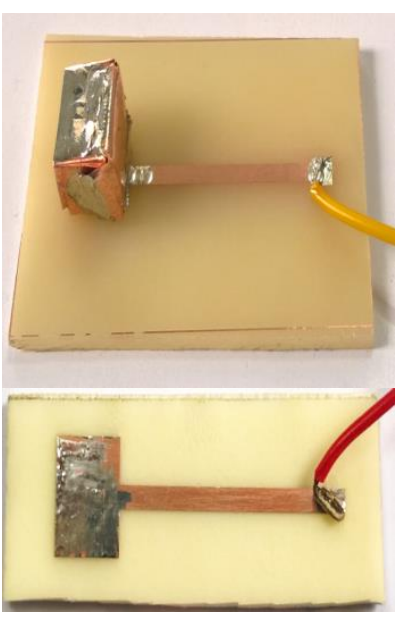

(a)

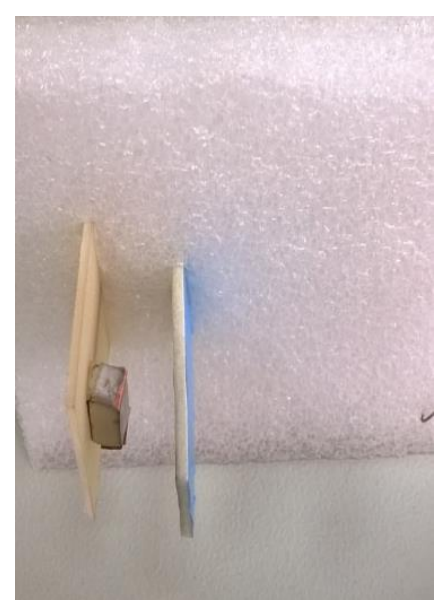

(b)
Fig. 2.2 (a) Fabricated Image of the negative positively feed based and negative feed cantilever substrate, (b) Image of mounted macor cantilever on a support. 


\section{B. Design \& Fabrication of Signal Detection Circuit and Interfacing}

The signal detection circuit for the flexible sensor array consists of a capacitance to voltage converter using ICCAV424. The input capacitance from sensor and ICs internal reference capacitance are compared and converted into DC voltage. Fig. 4.3 shows the schematic of converter IC. The measured output voltage from CAV424 circuit as shown in Fig. 2.4, will be given to the analog port of Arduino controller for voltage measurements.

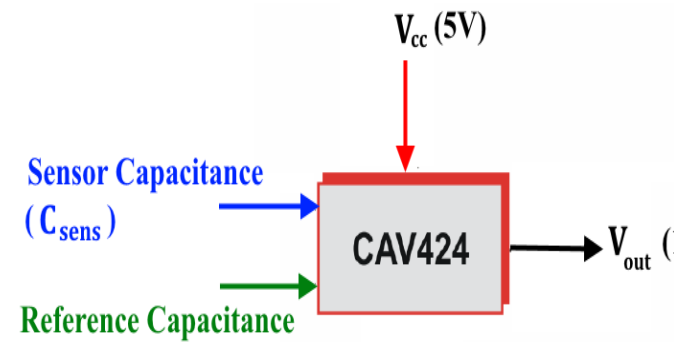

Reference Capacitance

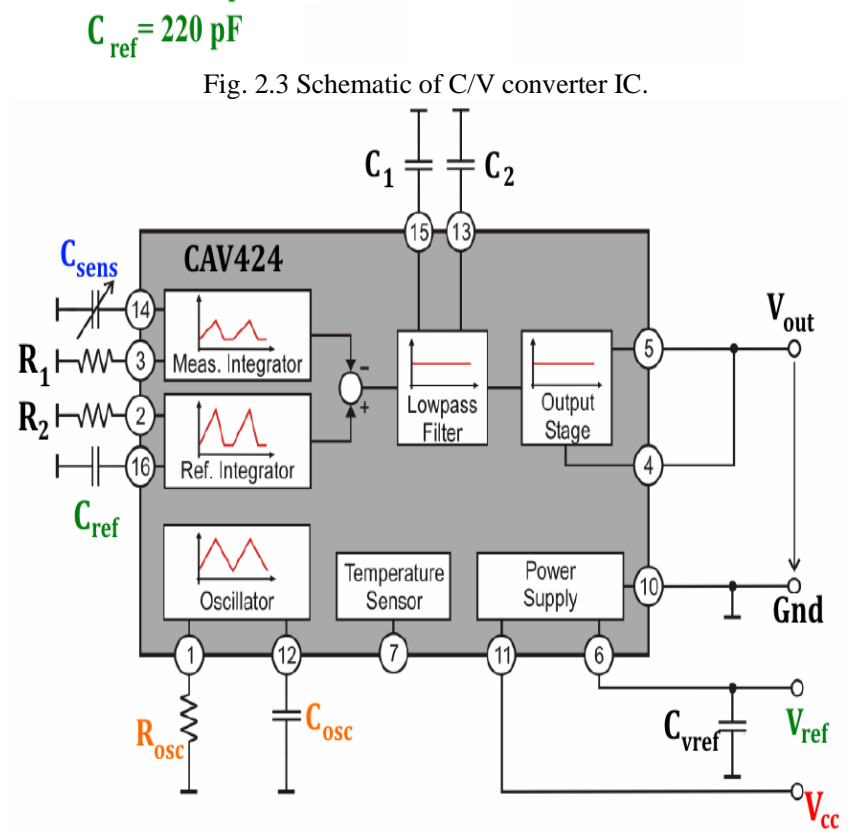

Fig. 2.4 Circuit Diagram for biasing CAV424 C-V converter.

The circuit is operated in differential capacitive input signal mode. In this configuration, the input capacitance ( $\left.C_{\text {sens }}\right)$ from the macro cantilever bending is compared with a reference capacitance $\left(C_{\text {ref }}\right)$ of $220 \mathrm{pF}$. Both capacitances are charged and discharged synchronously with a constant current by their separate integrator blocks. The charge currents for Csens and Cref can be adjusted using $R_{l}$ and $R_{2}$ respectively. The time constant for the charge and discharge process is given by the oscillator block, whose frequency can be adjusted using $C_{O S C}$ and $R_{O S C}$. At the low pass stage, the difference of these saw tooth output voltages is converted into a DC voltage.

The output voltage $V_{\text {OUT }}$ at pin 5 is ratio metric to the supply voltage and is a linear function of $1 /$ Csens. It can be referenced to GND or to pin 6 to obtain the differential output voltage $V_{D I F F}=V_{O U T}-V_{R E F}$. The typical values selected for biasing the circuit are discussed in Table 2.1
Table 2.1 Circuit value of CAV424

\begin{tabular}{|l|l|}
\hline Ciruit Component & Value \\
\hline$R_{1}$ & $560 \mathrm{k} \Omega$ \\
\hline$R_{2}$ & $600 \mathrm{k} \Omega$ \\
\hline$C_{\mathrm{ref}}$ & $220 \mathrm{pF}$ \\
\hline$R_{\mathrm{osc}}$ & $47 \mathrm{k} \Omega$ \\
\hline$C_{\mathrm{osc}}$ & $220 \mathrm{pF}$ \\
\hline$C_{1}$ & $3300 \mathrm{pF}$ \\
\hline$C_{2}$ & $2200 \mathrm{pF}$ \\
\hline$V_{\text {ref }}$ & $2.5 \mathrm{~V}$ \\
\hline$V_{c c}$ & $5 \mathrm{~V}$ \\
\hline$C_{\text {Vref }}$ & $10 \mathrm{nH}$ \\
\hline
\end{tabular}

Fig. 2.5 shows the image of the fabricated capacitance to voltage converter circuit. The PCB has been realized on FR-4 substrate. SMD and ceramic lumped components are used for biasing the circuit.

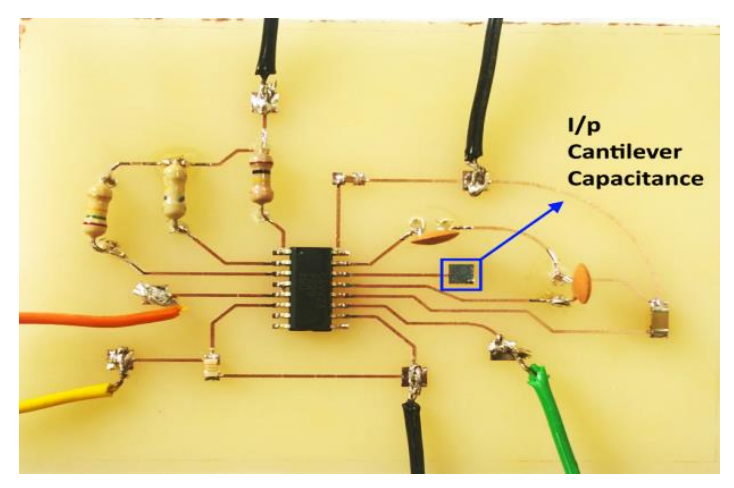

Fig. 2.5 Image of the fabricated C-V converter circuit.

\section{Development of Sensor System}

The fabricated $\mathrm{C}-\mathrm{V}$ converter circuit was connected to the necessary DC voltage source. The positive connection of the sensor is connected to the input capacitance pin (14) of the CAV424 and negative was commonly grounded with circuit grounded network.

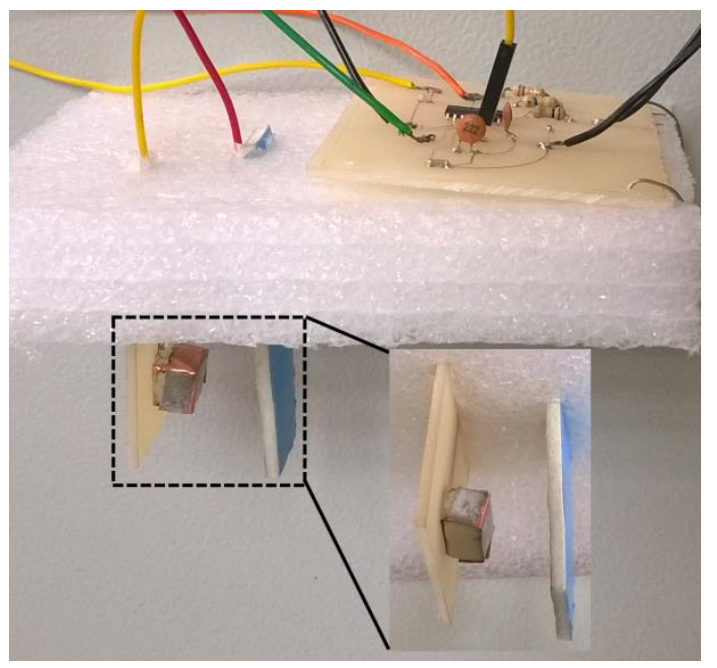

Fig. 2.6 Image of the assemble prototype macro cantilever capacitive sensor system. 
The output port $\mathrm{V}_{\text {out }}$ (Pin 5) was then connected to the analog port $\mathrm{A} 0$ of the Arduino controller for DC voltage measurement from the sensor system. Fig. 2.6 shows the complete setup of the capacitive sensor.

\section{RESULTS AND DISCUSSION}

The measured capacitance for variation in the distance between plates of the capacitive cantilever system is plotted in Fig. 4.7. In the fabricated sensor, the vibrational force from the metal joints of civil engineering structure bends the flexible macro cantilever for short interval of time and the position get restored again. For large vibration, the cantilever touches the +ve plate and short circuit condition results in zero capacitance. As the distance increase, the capacitance reduces in a linear manner.

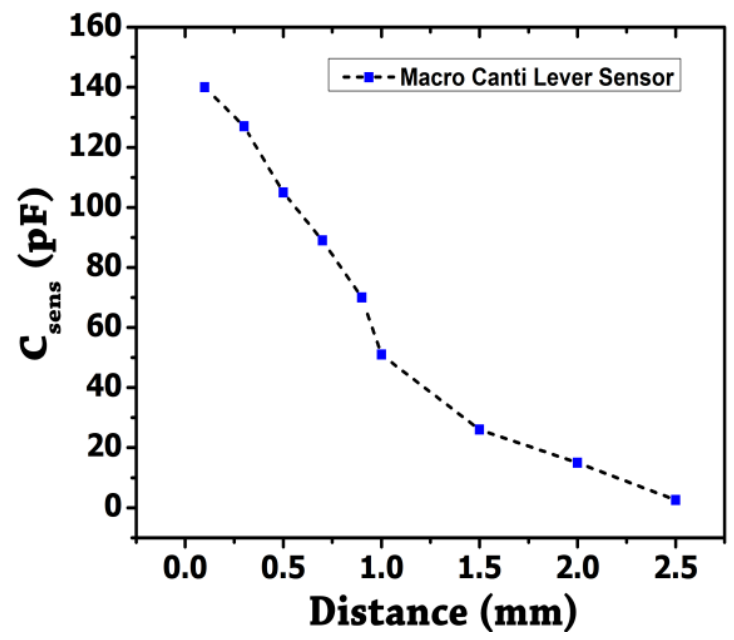

Fig. 3.1 Capactive response of macro canti level sensor

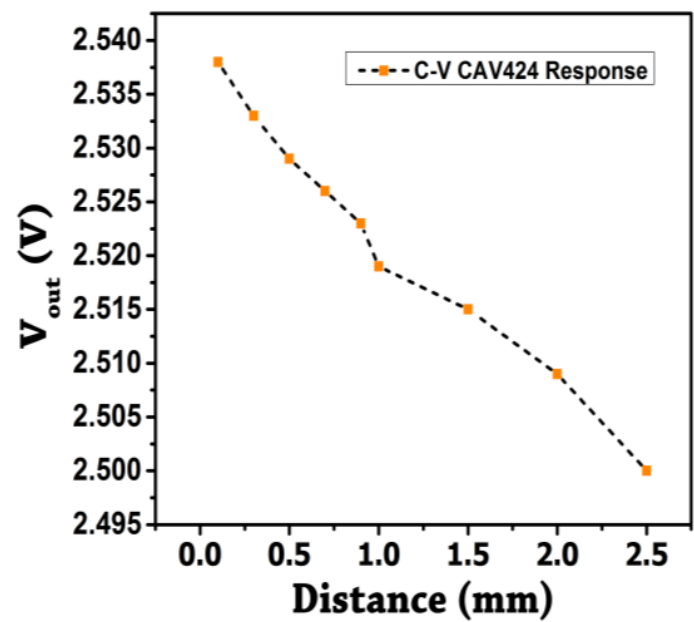

Fig. 3.2 Plot of the output voltage $\left(\mathrm{V}_{\text {out }}\right)$ vs. distance between the capacitive sensor plates.

The measured output voltage Vo from the prototype sensor shows a minor decrease in the DC level as the sensor capacitance reduces or distance increase. This is because the $\mathrm{C}_{\mathrm{ref}}$ is $220 \mathrm{pF}$. In order to obtain large variation in the output voltage, a higher value of $\mathrm{C}_{\text {ref }}$ is needed.
It can be observed that increase of the load on the sensor is the parameter of the stress on the structure. As the stress on the structure increases distance between positive and negative feed decreases. Distance is proportional to the capacitance or voltage. Thus by measuring the pattern of capacitance or voltage variation over a period of time and distance can co relate between stress on the structure and possible withholding capacity of the structure against the stress by identifying the failure point of civil materials.

Thus macro-cantilever based sensory studies prove to be very significant methodology for stress \& Vibration study in structural health monitoring (SHM).

\section{CONCLUSION}

A modified scaled up design of the capacitive macrocantilever unit-cell is developed and is used to realize a stress and vibrations in various civil engineering blocks. The proposed sensor shows a considerable change in the capacitance under the unit-cell where force distributions in extreme load conditions takes place on the civil structures. It is also indicative that three dimensional sensory applications can be built to an advantage.

\section{ACKNOWLEDGMENT}

This publication is an outcome of the R\&D work undertaken in the Project under the Visvesvaraya Ph.D. Scheme of Ministry of Electronics \& Information Technology, Government of India, being implemented by Digital India Corporation (Formerly Media Lab Asia). The authors also thank Sensor Laboratory, Electronics Engineering Department, SVNIT for providing the fabrication and testing facility.

\section{REFERENCES}

[1] J. Bungey, S. Millard, M. Grantham, Testing of Concrete Structures, $4^{\text {th }} E d$., New-York, NY, USA: Taylor and Francis, 2006.

[2] J. Brownjohn, "Structural heath monitoring of civil infrastructure," Philosophical Transactions of the Royal Society A: Mathematical, Physical and Engineering Sciences, vol. 365, no. 1851, pp. 589-622, 2007.

[3] J. S. Leng, D. Winter, R. A. Barnes, G. C. Mays, and G. F Fernando, "Structural health monitoring of concrete cylinders using protected fibre optic sensors," Smart Mater. Struct., vol. 15, no. 2, pp. 302, 2006.

[4] D. D. L. Chung, "Structural health monitoring by electrical resistance measurement," Smart Mater. Struct., vol. 10, no. 4, pp. 624, 2001.

[5] P. Giannelli, A. Bulletti, L. Capineri, "Multifunctional Piezopolymer Film Transducer for Structural Health Monitoring Applications," IEEE Sens. J., vol. 17, no. 14, pp. 4583-4586, 2017.

[6] H. Cao, S. K. Thakar, M. L. Oseng, C. M. Nguyen, C. Jebali, A. B. Kouki, J. -C. Chiao, "Development of Characterization of a Novel Interdigital Capacitive Strain Sensor for Structural Health Monitoring," IEEE Sens. J., vol. 15, no. 11, pp. 6542-6548, 2015.

[7] Y. Jia, K. Sun, F. J. Agosto, M. T. Quinones, "Design and characterization of a passive wireless strain sensor," Meas. Sci. Technol., vol. 17, pp. 2869-2876, 2007.

[8] R. Matsuzaki, A. Todoroki, "Wireless Flexible Capacitive Sensor Based on Ultraflexible Epoxy Resin for Strain Measurement of Automobile Tires", Sens. and Actuators A: Phys., vol. 140, no.1, pp. 32-42, 2007.

[9] Piyush N. Patel, Rahul Yadav, MeghayuAdhvaryu, "Design and Analysis of Diversified Mico-Cantilever Structure for Sensor Applicataion", 2nd IEEE International Conference on Emerging Technological Trends in Electronics, Communication and Networking, pp. 195, Dec-2014 